Proceedings

\title{
Pulse Wave Monitoring for Arterial Stiffness Detection Using a Simple Portable Tonometer ${ }^{\dagger}$
}

\author{
Gaëlle Lissorgues 1,* , Alexandre Bongrain 2, Lionel Rousseau 1, Nadia Madaoui 1, \\ Amandine Testi ${ }^{1}$, Laurie Valbin ${ }^{1}$, Sébastien Moussay ${ }^{2,3}$ and Pierre-Alexandre Chapon ${ }^{2,3}$ \\ 1 University Paris Est, ESYCOM-ESIEE, Bd Blaise Pascal, 93162 Noisy le Grand, France; \\ lionel.rousseau@esiee.fr (L.R.); nadia.madaoui@esiee.fr (N.M.); amandine.testi@edu.esiee.fr (A.T.); \\ laurie.valbin@esiee.fr (L.V.) \\ 2 Bodycap, 6 Rue De La Girafe, 14000 Caen, France; alexandre.bongrain@bodycap-medical.com (A.B.); \\ sebastien.moussay@bodycap-medical.com (S.M.); pierre-alexandre.chapon@bodycap-medical.com (P.-A.C.) \\ 3 UNICAEN, COMETE, INSERM, U1075, Normandie Université, 14032 Caen, France \\ * Correspondance: gaelle.lissorgues@esiee.fr; Tel.: +33-145-926-696 \\ + Presented at the Eurosensors 2017 Conference, Paris, France, 3-6 September 2017. \\ Published: 24 August 2017
}

\begin{abstract}
The work presented in this paper concerns the design, fabrication and test of a simple portable device able to measure in real time the cardiac frequency and the pulse wave, plus the pulse velocity in specific conditions (carotid and femoral locations). The sensing part is based on a thin film technology embedded in polymer and the data processing part is based on a classical pre-amplifying circuit associated with digitalization and shape detection algorithm. The whole device has been chosen as one demonstrator in the EveryWear project of the PROXIMA mission launched by the European Space Agency, the France's space agency CNES with space medicine specialists MEDES, to estimate the arterial stiffness variations under microgravity conditions and weightlessness. Raw data are stored through the remote tablet application and will be later processed using Matlab.
\end{abstract}

Keywords: pulse wave monitoring; arterial stiffness; portable medical sensor

\section{Introduction}

In recent years, a great emphasis has been placed on the role of arterial stiffness in the development of several cardiovascular diseases [1]. Carotid artery pulse pressure waveform is classically recorded by applanation tonometry [2], and its shape depends on the subject age, on the measurement position, and on the pathologies [3]. In this context, we developed a small portable device to simply monitor the pulse wave over repeated periods of time. The driving idea was to reduce the size of the sensor compared to gold standard tonometer, while keeping a sufficient sensitivity, suitable for a wide range of medical and sports applications. Effort was focused on the sensor design, fabrication and evaluation. Post data processing is then proposed and we can extract the augmentation index as the ratio between the augmentation pressure (being the height of the late systolic peak above the inflexion) and the total pulse pressure. In addition, this first sensor generation was selected to be tested during spaceflight conditions, which required some modifications in the sensor packaging and in the data transmission solution.

\section{Materials and Methods}

The operation principle of our tonometer is based on deformation measurement due to the pulse pressure transit in the artery using a piezoelectric thin film [4] and is conceived as a second skin solution. Indeed, the sensing unit is a disposable patch embedded in a polymer, while the 
electronic patch is hosting the power management, the microprocessor and memory, and the antennae in a reusable chip, Figure 1.

The sensor fabrication relies on AlN sputtering at low temperature (below $300{ }^{\circ} \mathrm{C}$ ). The AlN film characteristics were determined on test vibrating structures, giving the following piezoelectric coefficients: $\mathrm{d} 33, \mathrm{f}=3.6 \mathrm{pm} / \mathrm{V}, \mathrm{d} 31=21.8 \mathrm{pm} / \mathrm{V}$. XRD measurements were also performed to check the quality of the sputtered films, Figure 2. We obtain the AlN growth (with a rugosity around $3.5 \mathrm{~nm}$ ) directly on a Silicon substrate, with $C$ axis perpendicular to the substrate allowing a transverse and longitudinal coupling. The sensor response is then less dependent on the positioning.

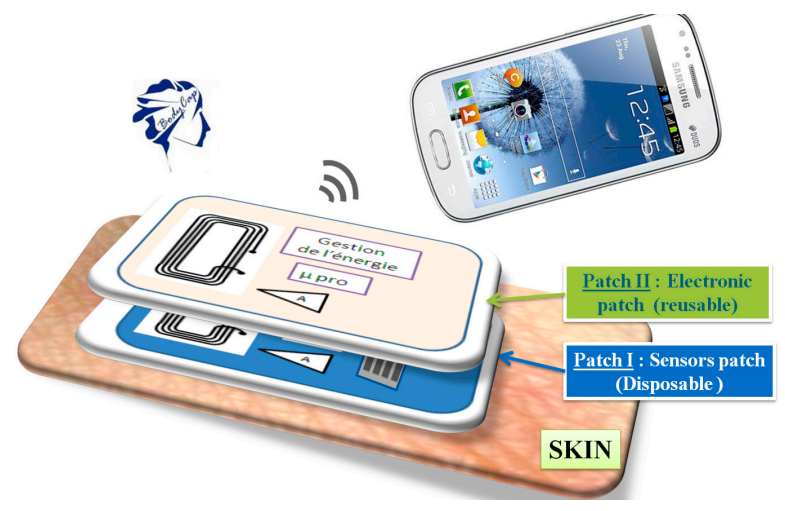

Figure 1. Principle of second skin concept.

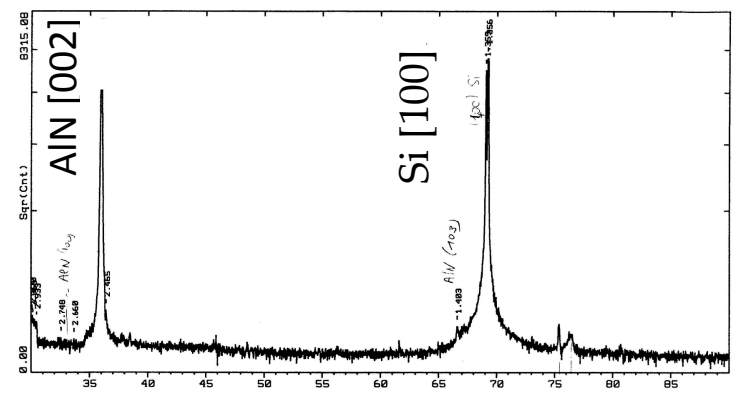

Figure 2. XRD measurement on fabricated AlN films.

Finally, we developed several designs with different shapes (circle, rectangular, etc.) to increase the sensitivity to deformation, directly related to the active surface, as shown on Figure 3 . We have then embedded the piezoelectric film into polymer film of thickness comprised between $20 \mu \mathrm{m}$ and more than $50 \mu \mathrm{m}$, both for better manipulation and reproducibility of the counter pressure applied with the finger tip. And we developed a specific package to fit the spaceflight constraints, leading to the evolutions presented in Figure 4.

\begin{tabular}{|l|l|l|}
\hline square & \multicolumn{2}{|c|}{ sectangle } \\
\hline Heart rate : \\
Mean : 63,69 pulses/min
\end{tabular}

Figure 3. Relation between sensor shape and sensitivity (higher noise with small surface)-the reference pressure was taken with a standard tensiometer as 98 Systolic, 68 Diastolic and 66 beat per minute on the left arm while our sensor was used on the right carotide. 
For monitoring and data processing, a control electronic and a Human Machine Interface (HMI) were developed. A charge amplifier stage and a 4-order low-pass filter for signal pre-conditioning compose the control electronic analog front end. The cut-off frequency of the filter was set at $25 \mathrm{~Hz}$. The signals are digitalized using a 10-bit Analog-to-Digital converter embedded in the microcontroller used (Atmega328p) and transmitted by serial communication to the interface. The HMI developed in this study allows monitoring, recording and post-processing of pulse wave sequences from the piezoelectric sensor, Figure 5. The post-processing is done off-line, and mainly consists in filtering (low pass at $12 \mathrm{~Hz}$ and high pass at 0.2 or $0.5 \mathrm{~Hz}$ ), and significant peak detection. The high pass filter is used for base line drift reduction. The low pass filter is used to suppress out-of-band noise (e.g., $50 \mathrm{~Hz}$ of the power supply) and also to smooth out the signal of interest.

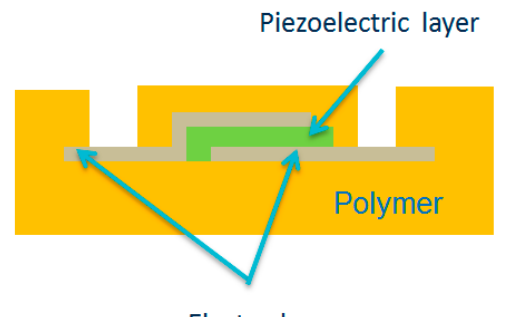

Electrodes

(a)

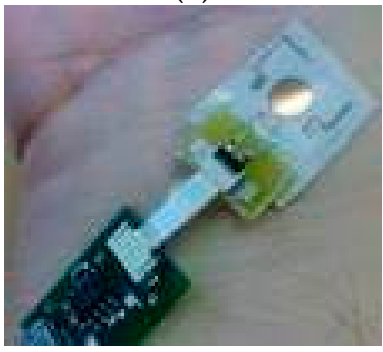

(d)

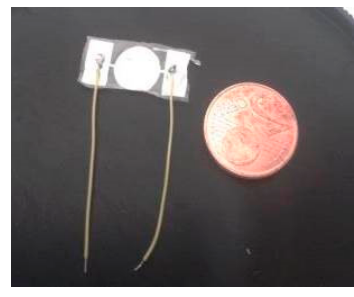

(b)

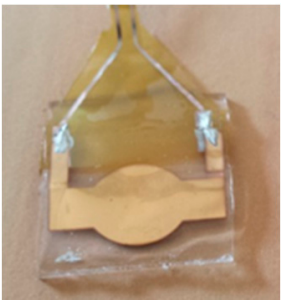

(c)

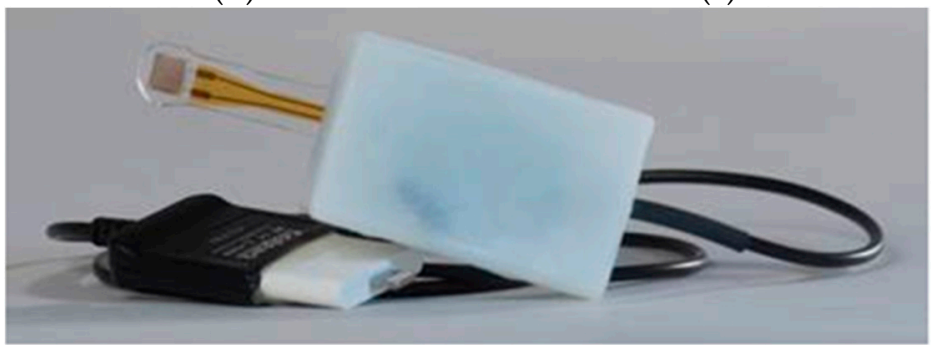

(e)

Figure 4. Sensor fabrication and evolutions; (a) side view; (b) first design; (c) second design embedded in parylene; (d) embedded with electronic circuits; (e) final version with a finger ring (C) images CNES.

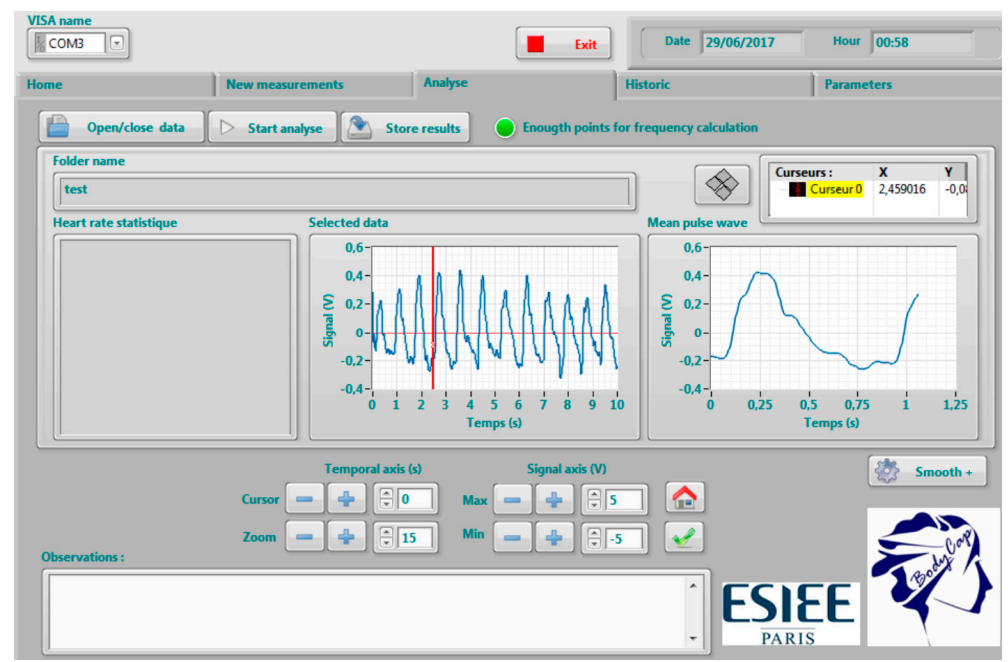

Figure 5. Example of the human machine interface developed to extract the main arterial characteristics.

\section{Results}

We fabricated several sensor prototypes which were the used for measurement on healthy volunteers, aged between 20 and 60 years old, male and female. First, a comparison with classical 
electrocardiogram (ECG) was done to both validate our measurements and offer the opportunity to synchronize the pulse wave form on a single reference, as illustrated on Figure 6.

Second, we have chosen the best sensor to collect more data and improve the signal processing, Figure 7. In order to determine the peaks of interest on each pattern of the pulse wave, the maximum on each wave is first determined. Then, using the second derivative as described in [5], we determine the wave foot, the end of the wave and the dicrotic wave position. The result is shown on Figure 8 for one pattern. The detected dots allow extracting cardiovascular parameters such as heart rate, systolic and diastolic cycles and the reflection index and reflection duration of the pulse wave directly related to arterial stiffness. After determining the foot of each wave, we will be able to determine the pulse wave velocity (PWV) from two measurements on two different locations (carotid-femoral). This requires either two simultaneous measurements (thus two sensors) or two measurements with the same sensor but with an ECG signal taken as reference for each measurement. The accurate PWV determination is one of the perspectives of this work.

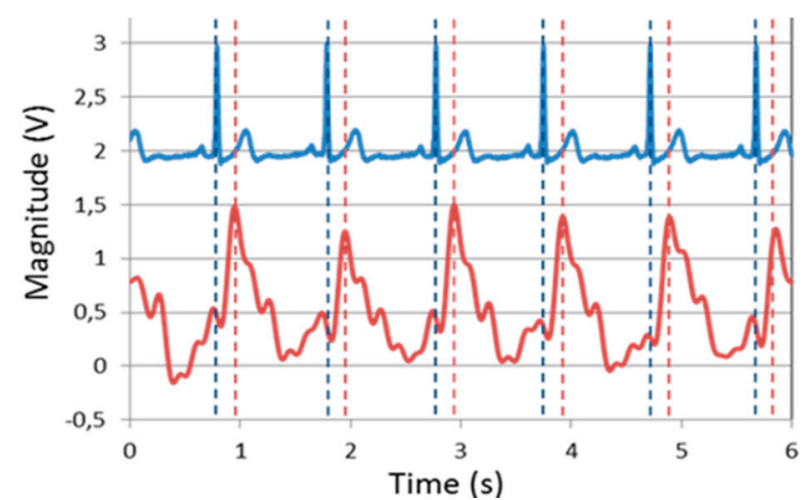

Figure 6. Comparison between classical ECG (blue) with our sensor (red) measuring the pulse wave.

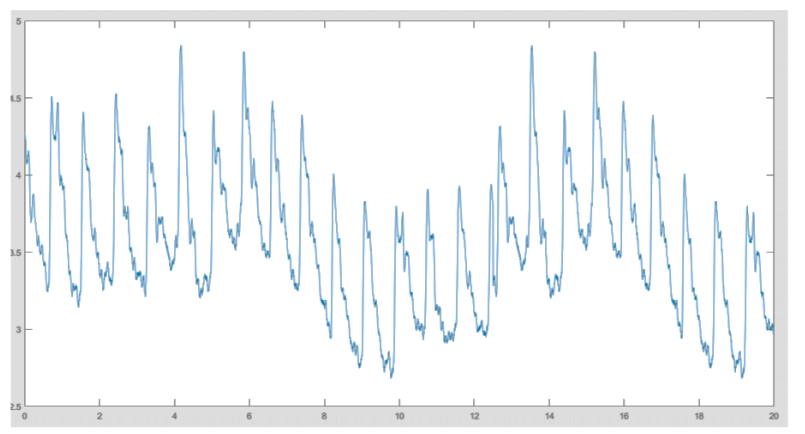

Figure 7. Raw data measurement performed with an improved sensor, before filtering.

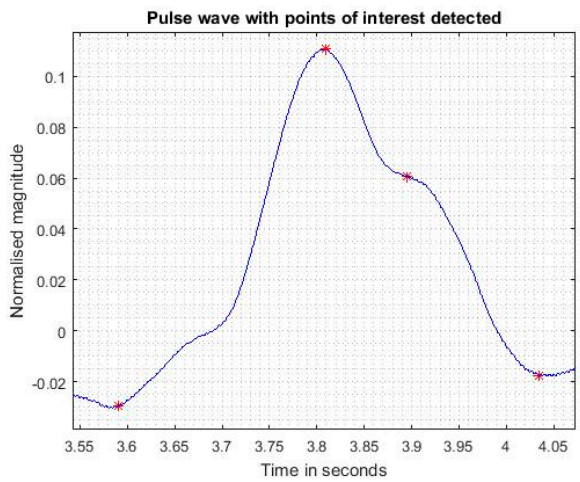

Figure 8. Pulse wave with points of interest detected. 


\section{Conclusions}

Arterial stiffness detection is of high interest in cardiology. An accurate measurement method used to extract the blood pulse wave with a simple small size system compared to medical tonometer was demonstrated. Further developments will focus on the pulse velocity calculation based on measurement done at two locations.

Acknowledgments: The authors wish to thank the ESA-CNES-MEDES and INSERM teams for their support to send the tonometer as a technological demonstrator in the International Space Station in 2016-2017.

Conflicts of Interest: The authors declare no conflict of interest.

\section{References}

1. Van Bortel, L.M.; Laurent, S.; Boutouyrie, P.; Chowienczyk, P. Cruickshank, J.K.; Backer, T.D.; Filipovsky, J.; Huybrechts, S.; Mattace-Raso, F.U.S.; Protogerou, A.D.; et al. Expert consensus document on the measurement of aortic stiffness in daily practice using carotid-femoral pulse wave velocity. J. Hypertens. 2012, 30, 445-448.

2. Yousefi Rizi, F.; Setarehdan, S. K.; Behnam, H.; Alizadeh Sani, Z. Study of the effects of age and body mass index on the carotid wall vibration: Extraction methodology and analysis. Proc. Inst. Mech. Eng. H 2014, 228, 714-729.

3. Kingsley, J.D.; Mayo, X.; Tai, Y.L.; Fennell, C. Arterial stiffness and autonomic modulation following free-weight resistance exercises in resistance trained individuals. J. Strength Cond. Res. 2016, 30, 3373-3380.

4. Bongrain, A.; Rousseau, L.; Valbin, L.; Madaoui, N.; Lissorgues, G.; Verjus, F.; Chapon, P.A. A New Technology of Ultrathin AlN Piezoelectric Sensor for Pulse Wave Measurement. Procedia Eng. 2015, 120, 459-463.

5. Nenova, B.; Llive, I. An automated algorithm for fast pulse wave detection. Int. J. Bioautom. 2010, 14, 203-216.

(C) 2017 by the authors. Licensee MDPI, Basel, Switzerland. This article is an open access article distributed under the terms and conditions of the Creative Commons Attribution (CC BY) license (http://creativecommons.org/licenses/by/4.0/). 